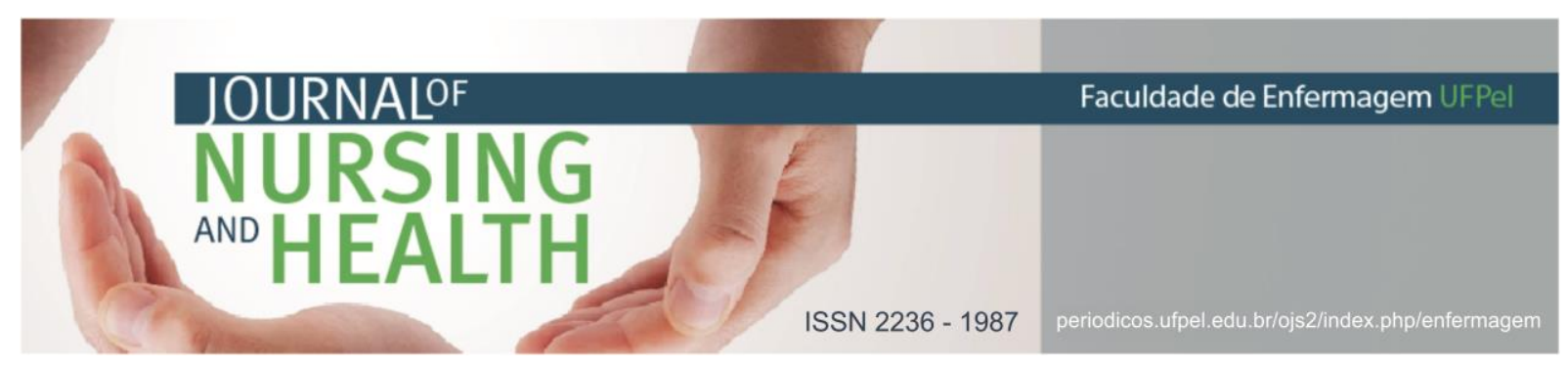

ARTIGO ORIGINAL

\title{
Experiencias y creencias de madres sobre la lactancia materna exclusiva en una región de México
}

\author{
Experiences and beliefs of mothers about exclusive breastfeeding in a region of \\ Mexico
}

\section{Experiências e crenças das mães sobre o aleitamento materno exclusivo em uma região do México}

Mota-Castillo, Pedro Javier ${ }^{1}$; Hernández-Ibarra, Luis Eduardo²; Pelcastre-Villafuerte Blanca Estela³; Rangel-Flores, Yesica Yolanda ${ }^{4}$

Cómo citar este artículo: Mota-Castillo PJ, Hernández-Ibarra LE, Pelcastre-Villafuerte BE, RangelFlores YY. Experiencias y creencias de madres sobre la lactancia materna exclusiva en una región de México. J. nurs. health. 2019;9(1):e199103

\section{RESUMEN}

Objetivo: explorar las experiencias y creencias sobre la lactancia materna exclusiva de madres que acuden a consulta nutricional a un hospital público. Método: se llevó a cabo un estudio cualitativo en la ciudad de Soledad de Graciano Sánchez, México. Participaron 31 madres lactantes en cuatro grupos focales. Se realizó análisis de contenido convencional. Resultados: las participantes reconocen la lactancia materna como la alimentación ideal para sus hijos, no obstante, señalan carencias de información en materia de lactancia y abogan por una orientación más completa y veraz. Diversas circunstancias comprometen el amamantamiento y hacen la lactancia poco placentera en términos de sensación física. Las mujeres resaltan el papel de la madre y abuela como principal fuente de apoyo e información a diferencia del personal de salud. Consideraciones finales: la educación en lactancia humana debe fortalecerse. El personal sanitario debe incrementar su papel y presencia para promover la lactancia materna exclusiva.

Descriptores: Lactancia materna; Investigación cualitativa; Salud materna.

\section{RESUMO}

Objetivo: explorar as experiências e crenças sobre a amamentação exclusiva de mães que chegam a um hospital público para consulta nutricional. Método: um estudo qualitativo foi realizado na cidade de Soledad de Graciano Sánchez, no México. 31 mães lactantes participaram de quatro grupos focais. A análise do conteúdo convencional foi realizada. Resultados: os participantes reconhecem a amamentação como a dieta ideal para seus filhos, no entanto, apontam a falta de informação sobre a amamentação e defendem uma orientação mais completa e verdadeira. Diversas circunstâncias comprometem a amamentação e tornam a amamentação desagradável em termos de sensação física.

1 Licenciado en Nutrición. Maestro en Salud Publica. Centro de Investigación en Sistemas de Salud, Instituto Nacional de Salud Pública (México). E-mail: pedromota_02@hotmail.com http://orcid.org/0000-0002-1199-1968 2 Licenciado en Psicología. Doctor en Ciencias de la Salud. Universidad Autónoma de San Luis Potosí (México). Email: eduardo_ibarra@hotmail.com http://orcid.org/0000-0002-3889-7533

3 Licenciada en Psicología. Doctora en Psicología Social. Centro de Investigación en Sistemas de Salud Instituto Nacional de Salud Pública (México). E-mail: blanca.pelcastre@insp.mx http://orcid.org/0000-0003-4755-1881 4 Licenciada en Enfermería. Doctora en Ciencias Sociales, Antropología y políticas públicas. Universidad Autónoma de San Luis Potosí (México). Email: yesica.rangel@uaslp.mx http://orcid.org/0000-0001-5673-6891 


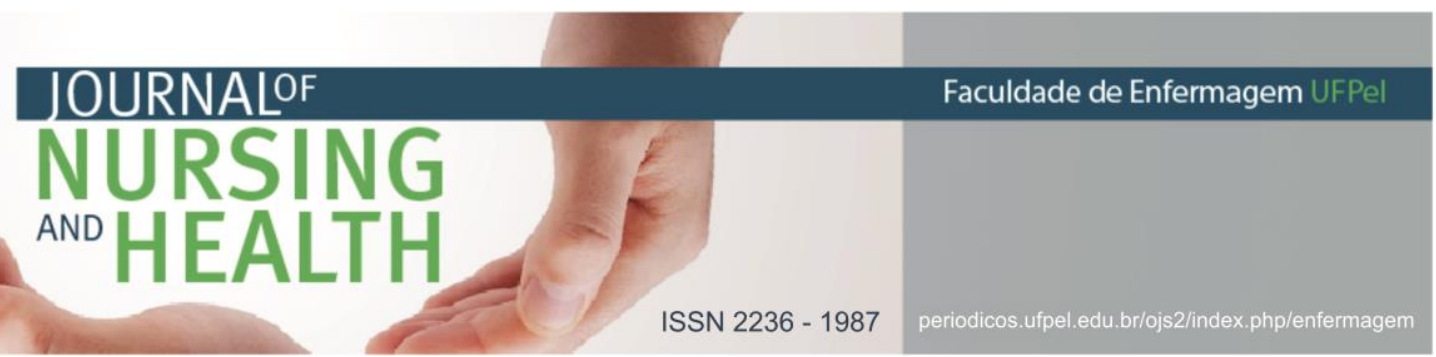

As mulheres destacam o papel da mãe e da avó como principal fonte de apoio e informação, diferentemente do pessoal de saúde. Considerações finais: a educação em lactação humana deve ser fortalecida. $O$ pessoal de saúde deve aumentar seu papel e presença para promover o aleitamento materno exclusivo.

Descritores: Aleitamento materno; Pesquisa qualitativa; Saúde materna.

\section{ABSTRACT}

Objective: explore the experiences and beliefs about exclusive breastfeeding of mothers who come to a public hospital for nutritional consultation. Methods: a qualitative study was carried out in the city of Soledad de Graciano Sánchez, Mexico. 31 lactating mothers participated in four focus groups. Analysis of conventional content was carried out. Results: the participants recognize breastfeeding as the ideal diet for their children, however, they point out the lack of information on breastfeeding and they advocate a more complete and truthful orientation. Various circumstances compromise breastfeeding and make breastfeeding unpleasant in terms of physical sensation. Women highlight the role of the mother and grandmother as the main source of support and information unlike the health personnel. Final consideration: the education in human lactation should be strengthened. Health personnel should increase their role and presence to promote exclusive breastfeeding.

Descriptors: Breast feeding; Qualitative research; Maternal health.

\section{INTRODUCCIÓN}

La leche materna es el alimento ideal para el recién nacido durante sus primeros meses de vida, esto debido a su contenido nutricional, hormonal e inmunológico, además representa la mejor fuente de lácteos durante los primeros dos años de vida. ${ }^{1}$ Debido a lo anterior, la lactancia materna exclusiva no sólo representa la práctica de alimentación idónea en los primeros seis meses de vida, sino una de las estrategias costo-efectivas más importantes en la prevención de la morbilidad y mortalidad infantil, ya que si esta práctica se universalizara, se estima se podrían evitar 823,000 muertes infantiles y 20,000 de mujeres de manera anual aunado a un ahorro económico de $\$ 300$ mil millones de dólares; $^{2}$ lo cual hace que su protección, promoción y apoyo sea un objetivo de primer orden para la salud pública.

Según cifras de la Encuesta Nacional de Salud y Nutrición
(ENSANUT) 2012,3 la práctica de lactancia materna muestra un deterioro evidente; en menores de seis meses el porcentaje de lactancia materna exclusiva tuvo una disminución de $22.3 \%$ a sólo $14.5 \%$ en 6 años, en el área rural dicha práctica descendió a la mitad de un $36.9 \%$ en 2006 a $18.5 \%$ en 2012. Las madres que nunca amamantaron a sus hijos mencionan en la ENSANUT una serie de razones o causas para no hacerlo, entre las cuales destacan "no tuve leche" $37.4 \%$, enfermedad en la madre con un $13.7 \%$, "el bebé no quiso" $11.4 \%$, causas que sugieren desconocimiento, además de poco o nulo apoyo por parte del personal de salud durante el inicio de la lactancia materna.

En el estado de San Luis Potosí, las cifras mostraron que $56.1 \%$ de los recién nacidos tuvieron un inicio temprano de la lactancia materna; a nivel nacional el porcentaje de lactancia materna ha mostrado un 


\section{ISSN 2236 - 1987}

aumento considerable, al pasar de $14.4 \%$ en 2012 a $30.8 \%$ en $2015,{ }^{3}$ sin embargo, sigue estando en los últimos lugares de lactancia materna en Centroamérica y el Caribe ${ }^{1}$ y dista de la recomendación realizada por la Organización Mundial de la Salud.

A pesar de estas cifras alarmantes, pocos trabajos se han preocupado por explorar y conocer las voces de las madres a quienes son dirigidas las estrategias de fomento a la alimentación al seno materno para intentar comprender las razones y argumentos que motiven o desincentiven esta práctica; y den pie a estrategias diseñadas acordes a la población blanco; por ello este estudio tuvo como objetivo explorar las experiencias y creencias sobre la lactancia materna exclusiva de madres que acuden a consulta nutricional a un hospital público de segundo nivel de atención en la mancha urbana de San Luis Potosí.

\section{MATERIALES Y MÉTODOS}

Se realizó un estudio cualitativo con enfoque fenomenológico, en el municipio de Soledad de Graciano Sánchez, San Luis Potosí, México. Utilizando un muestreo teórico, ${ }^{4}$ el principal que fueran madres de niños que tuvieran máximo un año de edad y se encontraran lactando. Participaron 31 madres que acudían a consulta nutricional de apoyo continuo en lactancia materna, el número de participantes se decidió según el principio de saturación temática.

La información fue obtenida en los meses de marzo a junio del 2014 mediante cuatro grupos focales. Fue utilizada una guía que contenía temas relacionados a sus experiencias, creencias y prácticas en torno a la lactancia materna exclusiva.

Las entrevistas y grupos focales fueron audiograbados y transcritos en un procesador de textos, posteriormente se realizó una lectura general del corpus para explorar el discurso de las madres, los temas emergentes y su importancia. Luego de esto se llevó a cabo una lectura línea por línea con el fin de generar los códigos conceptuales y vivos para posteriormente generar categorías $y$ validarlas en un proceso continuo y reflexivo.

La participación fue voluntaria y bajo consentimiento informado. Se atendieron los principios de respeto a la autonomía, autodeterminación y confidencialidad de las participantes. El estudio fue avalado por los comités de ética de la Facultad de Enfermería y Nutrición de la Universidad Autónoma de San Luis Potosí obteniendo la clave de registro CEIFE-2013-080 y del Hospital General de Soledad otorgándole el folio de identificación CEI-HGS-002-14.

\section{RESULTADOS}

Se trabajó con mujeres en periodo de lactancia, que asistían a consulta nutricional en el hospital antes mencionado, con un rango de edad de los 16 a los 35 años, una edad promedio de 22 años, multíparas, en su mayoría con secundaria terminada. En cuanto a su situación laboral, casi en su totalidad, se dedicaban únicamente a las labores del hogar. 


\section{Motivos para amamantar}

Las participantes del estudio, reconocen a la lactancia materna como el alimento ideal para sus bebés $y$ valoran de manera positiva la práctica, comentan como motivos principales para iniciar la alimentación al pecho: el bienestar de sus hijos, su sano desarrollo, además de que al ser un proceso meramente natural $y$ fisiológico, disminuye el gasto familiar en gran medida, esto lo describen como otra de las bondades de la lactancia materna, el ahorro que significa el prescindir de la compra de fórmulas infantiles o biberones, insumos propios que caracterizan una alimentación artificial

[...] pues que es mejor, la mayoría de las personas que a mí me comentaron de lo del niño fue [el beneficio de] la economía ¿no?, ahorita no vas a gastar en leche más que en tu pecho y el único gasto que vas a tener, pues es lo pañales y eso pues si más que nada la economía [...]. (Mujer 1, Grupo focal 4)

[...] es que es más sana [la leche materna], son las primeras vacunas que uno le ofrece al niño... uno no tiene el gasto para comprar, para darle la leche [de fórmula], nos ayuda a nosotros las mujeres en varios aspectos y en lo personal a adelgazar. (Mujer 2, Grupo focal 4)

\section{Otra manera de decir te quiero}

Otra de las cuestiones que mencionan en sus discursos es la sensación de bienestar para con su bebé, lo cual, desde su punto vista, fortalece la relación madre e hijo y aluden a que es una de las mejores maneras para demostrarle su cariño al pequeño y crear un lazo de comunicación afectiva con él. Mencionan además que la experiencia complementa y es una extensión de cuando llevaban en el vientre su hijo/a, y lo expresaron de la siguiente manera:

[...] bueno a mí [me motivó] para crear ora si como el lazo, ¿no?, de sentirla a ella, y pues sí, para que sepa que la quiero [...] (Madre 1, Grupo focal 3)

[...] pues la experiencia que se siente al amamantarlo, sentir que estás junto a ellos, así como cuando los llevas en tu panza [...]. (Madre 1, Grupo focal 2).

\section{Lactancia materna versus fórmula infantil}

A diferencia de lo que se cree, las madres que participaron en el estudio conocen y señalan las ventajas que tiene el amamantar a diferencia de la fórmula infantil, resaltan los beneficios que trae consigo el dar pecho, refieren la disminución de la probabilidad de desarrollar cáncer cervicouterino $\mathrm{y} / \mathrm{o}$ de mama, la pérdida de peso o el pronto regreso a su peso normal, por citar algunos de los beneficios para la madre y el mejor desarrollo del menor en diversos ámbitos. Prueba de ello el siguiente comentario:

[...] yo por lo que yo sé, puede ser que también sea un método anticonceptivo, ayuda a que la 
mujer tenga menos riesgo de contraer cáncer, es más saludable para los niños, tengo entendido que está comprobado que ayuda a estimular a desarrollar más rápido el ser sociable [...] (Mujer 1, Grupo focal 4).

Otro de los aspectos que resaltan las madres, es el rol protector que juega la lactancia materna frente a diversas enfermedades, la mayoría conocen este hecho, y algunas otras de manera empírica se han ido dando cuenta de ello, ya que refieren verse motivadas por el estado de salud que presentaron sus anteriores hijos alimentados al seno materno y lo confirman al equiparar la salud que presentaban a la misma edad los alimentados al pecho contra quienes se les ofreció sucedáneo de leche humana y se dan cuenta de las ventajas y beneficios.

[...] pues más que nada la salud para los niños... pues en mi caso no se me ha enfermado, si acaso una gripilla o así, pero que se me haya enfermado nunca, y este pues yo pienso que sí [...] (Mujer 2, grupo focal 3).

[...] pues a mi primer bebé yo no le di pecho y a ella sí [bebé reciente], y pues mi primer bebé sí es muy enfermizo y a ella hasta ahorita va a cumplir seis meses y no se ha enfermado [...] (Mujer 1, grupo focal 4)

\section{Apoyo familiar en la etapa de lactancia}

Según las experiencias y prácticas de las participantes, sus madres y abuelas tienen un papel importante durante la lactancia, no sólo fomentan y motivan a sus hijas o nietas con sus propias experiencias, también reafirman la importancia de que el bebé sea alimentado con seno materno durante los primeros meses de su vida. Las participantes también dan testimonio de cómo sus madres reconocen que el mejor alimento para los bebés es la leche materna, por lo cual impulsan a que continúen con dicha práctica brindándoles el apoyo necesario que va desde la motivación verbal hacia la alimentación a libre demanda del bebé hasta la realización de masajes o preparación de bebidas para que su leche sea de mejor calidad para él menor. Lo anterior así lo describen un par de madres participantes,

[...] a mí, mi mamá me platica que es mejor darles pecho, porque es más saludable y la leche sale ya calientita, para ya no calentar la leche y aparte que le ayuda también a uno, pues para tener su peso [...] (Mujer 3, Grupo focal 4).

[...] A mí por ejemplo mi mamá me dice que eso es lo importante para que ellos estén bien, yo le digo que no puedo y ella me da pues, masajes, ella es la que me está ayudando ahorita y pues mi esposo pues también me dice tu échale ganas no te desesperes, porque como le digo ya me desesperé porque no me sale y 
dice no, tu pégatela tu pégatela, pero, mi mamá es la que más me apoya [...] (Mujer 3, Grupo focal $3)$.

\section{No todo es miel sobre hojuelas}

Las madres que participaron en este estudio aluden a diversas dificultades y complicaciones por las que suelen pasar al amamantar a sus hijos, en ocasiones las grietas en los pezones, el dolor en los senos debido a la excesiva producción de leche, la dificultad para que "baje" la leche e inclusive el convivir con la idea de que el llanto es debido a que su bebé no llena con la leche que ellas le ofrecen, constituyen sólo algunas de las razones más comunes por las cuales esta práctica se ve comprometida y no se lleve a cabo como lo indican las recomendaciones de diversos organismos de salud a nivel nacional o mundial, problemas derivados en su mayoría de un mal agarre al pezón. Así lo describe una de las participantes:

[...] como casi no me salía [leche], y me empezó a lastimar [el pezón], entonces a mi si me agrietó y hasta me sangraba y si también batallé y si también se me puso malita, si también de donde no comía lo suficiente, entonces ya ahorita le empecé a completar con la formula [...] (Mujer 3, Grupo focal 1).

\section{Los cambios que trae consigo brindar lactancia materna}

Las madres hablan acerca de un sacrificio en las actividades diarias por el hecho de estar amamantando a un niño, de manera más marcada las que refieren llevar la práctica a libre demanda o bien ofreciéndole tomas a su bebé cada dos o hasta tres horas, en su discurso aparecen: la descompensación en cuanto a las horas de sueño, el no tener tiempo de realizar ejercicio, o algunas labores domésticas, aunque la responsabilidad de tener bien alimentado a su bebé se vuelve prioridad sobre esas actividades, como lo dejan ver en las siguientes citas:

[...] a lo mejor hacer el quehacer, bueno, yo que estaba acostumbrada a hacer el quehacer así seguido, y ahorita ya me tengo que parar y en lo que voy y le doy de comer y luego ya sigo y luego otra vez [...] (Mujer 2, Grupo focal).

[...] dormir también, yo cada rato, como a cada rato quiere estar comiendo, entonces a cada rato me estoy levantando para darle de comer, casi como cada hora o a lo mucho dos horas [...] (Mujer 5, Grupo focal 1).

\section{La primera vez lactando}

En el caso de las mujeres primíparas, los miedos y el desconocimiento menguan la práctica de la lactancia materna, circunstancias como: el no conocer cuánto es lo que está consumiendo o si lo que el niño está consumiendo es suficiente, son frecuentes en el discurso de las mamás participantes y en ocasiones genera mucha incertidumbre y temor de que algo no está bien o que algo están haciendo mal. Dichos temores fungen como impedimentos para el desarrollo 


\section{JOURNAIOF

de la alimentación al pecho, así lo expresó una de las entrevistadas:

\section{[...] a mi todo me sorprendió,} como también es la primera, uno no sabe, uno tiene miedo, uno se desespera, de que no le sale leche luego, luego, uno cree que en cuanto nace el bebé instantáneamente te va a salir un chorro de leche y no es así, uno dice y ¿por qué no me sale? a lo mejor soy yo, estoy mal, nunca me va a salir, ¿qué voy a hacer?, y uno se preocupa mucho porque se ve la diferencia, se ve mucho la diferencia cuando no le dan pecho y cuando si le dan [...] (Mujer 1, Grupo focal 3).

\section{Lo que dice la abuela}

Según lo encontrado, nos percatarnos de mitos que aún siguen latentes entre las madres, los cuales viajan de manera generacional por medio de las voces de las madres y abuelas de las participantes, además de que el charlar con amigas y vecinas los complementan y refuerzan. Exacerban el cubrirse ciertas áreas del cuerpo, entre las más importantes la espalda y la cabeza, mencionan que de no hacer esto, su producción de leche cesará, o bien al ingerir alimentos secos como el camote puede llegar a que como ellas lo refieren "se seque el pecho", otra de las causas para que suceda lo anterior es el consumir alimentos a bajas temperaturas 0 exponerse a ellas, así lo describen un par de madres:

[...] la gente que me ve me dice, es que te tienes que tapar porque si no, no te va a bajar la leche, te vas a tener que tapar, tienes que enredarte mucho los pechos para que produzcas mucho, que porque te enfrías, eso es lo más que me llegaba a decir la familia de él o que me llegaron a decir de antes fue eso, que si uno no se tapaba y eso se iba la leche [...] (Mujer 2, Grupo focal 4).

[...] pues se puede ir por corajes o por sustos o pos gustos, o porque no te cubras la espalda [...] (Mujer 3, Grupo focal 4).

Para intentar aumentar la producción de leche, a la mayoría de las mamás se le les ha recomendado durante la etapa de lactancia, el consumo de bebidas alcohólicas, más frecuentemente la cerveza, así como atoles, tés, o infusiones calientes. Esto con la finalidad de que la leche "baje" más rápido o bien que sea "más gruesa", lo cual quiere decir que sea de mejor calidad. Aunque en algunos casos la experiencia de ellas mismas probando dichos remedios caseros, desmienten las aseveraciones de sus allegados.

[...] A mí me habían dicho que el atole de garbanzo, el de masa y el que es parecido al pulque, el aguamiel, si con ese [sale más leche], bueno me dijeron, yo nunca lo tomé porque no me gusta, los otros tres si, y la verdad no, no funciona [...] (Mujer 5, Grupo focal 4).

\section{Por una orientación más completa}

Las madres indican, que dentro de las actividades que llevaban a cabo 


\section{JOURNAIOF

en su centro de salud, la orientación y educación en lactancia humana era parte de esto, algunas con sesiones, algunas otras con una sola plática, pero la mayoría de ellas coinciden en que se les hablaba, pero no lo suficiente de las directrices de la alimentación al seno.

[...] pues en el centro de salud, fue donde más me dijeron que ahora si lo mejor que podía darle era darle pecho, para eso, para como esta chiquita como es pura, ahora si, no lleva grasa y no lleva sal ni nada entonces era para reforzarle sus defensas, su primer alimento [...] (Mujer 3, Grupo focal 2).

\section{Del dicho al hecho}

Las mujeres demandan una orientación más verídica, más apegada a la realidad que pueden enfrentarse una vez que hayan decidido amamantar, a la par las madres que participaron en el estudio refieren que no es un acto del todo placentero, ya que suele haber complicaciones y experiencias no agradables que durante las sesiones informativas acerca de amamantar se omiten, dirigiendo la asesoría específica para esa etapa dentro de un contexto sublime y utópico, además de que dicha orientación debe ser realizada por personas que no sólo ya hayan experimentado la sensación de ser madre sino también la de haber alimentado al pecho.

[...] a mí con mi primer niño las pláticas me dijeron "le va a hacer muy bien al niño, que va a ser más fácil, usted nada más se va a voltear si el niño está llorando y le va a dar pecho", o sea todo te lo pintan muy bonito, pero no te dicen-te va a doler, mira, si no le vas a dar pecho al niño te vas a chorrear ahí a media calle- o así o sea nada de eso te platican y yo quiero pensar que alguien que te da una plática ha tenido un niño o conoce a alguien que ve lo que pasa y esas partes que se podrían decir un poco feas, no las dicen. (Mujer 2, Grupo focal 4).

[...] Igual si de que si no te baja rápido [la menstruación] a mí no me dijeron y yo si me asusté como dos meses porque no me bajó y yo pensé que estaba embarazada y ya platiqué con mi mamá y me dijo, -no, ¿apoco no te habían dicho que mientras des pecho no te va abajar? [...] (Mujer 1, Grupo focal 4).

\section{DISCUSIÓN}

El presente estudio tuvo como objetivo conocer las experiencias y creencias en torno a la lactancia materna de madres que asisten a un hospital público en San Luis Potosí, México.

La lactancia materna ha sido reconocida por diversas organizaciones como el alimento exclusivo para los bebés durante sus primeros seis meses de vida y de manera complementaria, no sólo dentro del rol nutricional que juega al proveer al bebé de las cantidades específicas de leche que necesita, ${ }^{5}$ sino a nivel inmunonutricional al coadyuvar a la maduración de su sistema inmune. ${ }^{6}$ 


\section{JOURNALOF

En primer lugar, las madres aluden a la alimentación al pecho como un protector del bolsillo familiar, ya que se ha reportado un promedio de 30 latas de fórmula infantil para mantener una lactancia artificial durante los seis primeros meses de edad del infante lo que se traduce en un costo entre los 754 y los 801 dólares. ${ }^{7}$ Cabe mencionar que en estos datos no se incluyen los derivados de las consultas al pediatra por afecciones gastrointestinales, y de vías respiratorias asociadas al consumo de formulas.

Las mamás refieren también un bienestar y una sensación agradable al amamantar a sus hijos, el acercamiento que tiene el pequeño con la madre proporciona al niño el confort de la cercanía además de que lo dota de seguridad, lo que corresponde a lo reportado en otros estudios al señalar que la lactancia materna y el buen apego favorece no sólo los lazos afectivos entre madre e hijo, sino también su desarrollo psicomotor y la salud óptima del niño. Circunstancia también comentada en otros trabajos. ${ }^{8-9}$

Las madres comentan de igual manera que ellas notan cómo la leche fortalece el sistema inmune de sus hijos haciéndolos más fuertes y resistentes a infecciones, beneficios percibidos por ellas, esta apreciación coincide con lo reportado anteriormente al indicarse que la leche materna es la responsable de mantener el nexo inmunológico madre-hijo, al suministrarle a éste elementos protectores, células y factores solubles en su mayoría, los cuales en el neonato están ausentes, situaciones similares en cuanto a la protección en un amplio rango de enfermedades, sobretodo infectocontagiosas, lo que concuerda con trabajos reportados en poblaciones semejantes ${ }^{10-11}$ y que forma parte del discurso con el que el personal médico cree convencer a las madres para que inicien y mantengan esta práctica. ${ }^{12}$

Las participantes nombran a la familia en su discurso como un promotor importante para comenzar y continuar con la práctica situación observada en otro estudio ${ }^{13}$ lo que indica una aceptación total hacia la lactancia materna como la mejor y más sana opción. También se destaca el papel de la familia, en particular, de la pareja, ya que el incluirla en la educación prenatal se ha asociado con mayores tasas de intención de amamantar de manera exclusiva. ${ }^{14}$

En nuestro estudio, las madres de las entrevistadas suelen ser las defensoras de la práctica, circunstancia similar a lo reportado por otros autores ${ }^{15-16}$ mencionan que esta figura realiza un papel clave en el fomento, aunque, diferente a lo encontrado en nuestro estudio, también influye en la obstaculización de la práctica. Esta diferencia pudiera deberse a que quienes participaron en este estudio eran madres que acudían a atención nutricia, asunto que podría situarlas entre el grupo más proactivo y sensibilizado con el tema de la lactancia materna exclusiva.

En cuanto a las madres primíparas, el desconocimiento y la incertidumbre se suman a las dificultades de esta nueva etapa comprometiendo el mantenimiento de la lactancia materna, situación similar a lo observado en estudios 8,17 que 


\section{JOURNALOF \\ NURSING \\ ANO HEALTH}

ISSN 2236 - 1987

necesario contar con profesionales de la salud sensibilizados, comprometidos, convencidos y debidamente capacitados sobre amamantamiento, asunto que empieza a ser valorado en otro estudio. ${ }^{22}$

Dentro de este trabajo podemos darnos cuenta que las madres conocen los beneficios de la lactancia materna y la aprueban en su mayoría, aunque no suelen llevarla como manifiestan querer y como se les recomienda debido a la desesperación e incertidumbre que conlleva la poca información, capacitación y preparación que reciben, sobre todo al estar frente a la experiencia de ser madre por primera vez y es que confiesan su miedo al no saber si el bebé en realidad está comiendo, si su leche es de calidad, cuánta es la cantidad que están produciendo, entre muchas otras dudas y preguntas frecuentes acerca de este proceso, que debieran ser abordadas en la educación que se les brinda por parte del personal de salud.

\section{CONSIDERACIONES FINALES}

Con base en los hallazgos de este trabajo podemos concluir que los profesionales de la salud no están alentando de la mejor manera la lactancia materna exclusiva, ya que, según las madres que participaron, no se les brinda a las madres una capacitación de calidad que desmitifique prácticas ligadas a la mejora o bien al cese de la producción de leche en las madres. Contribuir a diluir dichos mitos debe ser parte importante de la orientación brindada por el personal de salud, ya que es 


\section{JOURNALOF \\ NURSING \\ M०HEALTH}

ISSN 2236 - 1987

impostergable para la salud pública el romper con los círculos donde se repite información poco útil para quienes pretenden brindar lactancia materna exclusiva por lo cual la educación en ellas es de suma importancia.

La educación en materia de lactancia humana, debe brindarse a las madres pero también a los padres de familia quienes deben asumir su responsabilidad durante esta etapa ya que influyen fuertemente en la decisión de su pareja de amamantar o no; esta educación, también debe ser primordial en el profesional de salud, ya que la mayoría de las complicaciones que suelen presentar las madres pueden ser totalmente prevenibles asegurándole y informando sobre un buen agarre por parte del niño al pecho, entre otras buenas prácticas.

Los hallazgos aquí presentados evidencian áreas de oportunidad en la promoción de la lactancia materna exclusiva, que sin duda alguna representa uno de los pendientes de mayor relevancia de la salud pública en México.

El presente trabajo reconoce la importancia de seguir realizando investigación en el tema, para explorar a profundidad las necesidades y demandas por parte de los profesionales de salud, para conocer de viva voz las barreras, dificultades, u obstáculos a los que se enfrentan o se hayan enfrentado para cumplir con la promoción y fomento de la lactancia materna exclusiva, lo cual nos ayudará a comprender de manera más amplia la situación actual de lactancia materna desde la óptica de los profesionales de la salud y de esta manera incidir en la creación de nuevas políticas públicas y programas, o bien modificar de manera más atinada los actuales y conseguir los objetivos que se planteen en materia de lactancia materna.

\section{AGRADECIMIENTOS}

A las madres que participaron en el estudio por brindarnos su tiempo y compartir sus experiencias. A los lectores y revisores anónimos del trabajo por sus valiosos comentarios a versiones anteriores.

\section{REFERENCIAS}

1 Victora CG, Bahl R, Barros JD, Franca GVA, Horton S, Krasevec J, et al. La lactancia materna en el siglo XXI: epidemiología, mecanismos y efectos a los largo de la vida. Lancet [Internet]. 2016[citado 2018 nov 29]387:1-16. Disponible en: http://www.incap.int/index.php/es/ publicaciones/publicacionesconjuntas-con-otrasinstituciones/doc_view/782-thelancet-serie-sobre-lactancia-materna2016-espanol

2 Gillespie S, Haddad L, Mannar V, Menon P, Nisbett N, et al. ¿Por qué invertir y qué se necesita para mejorar las prácticas de lactancia materna? Lancet [Internet]. 2016[citado 2018 nov 29];387:17-30 Disponible en: http://www.incap.int/index.php/es/ publicaciones/publicacionesconjuntas-con-otrasinstituciones/doc_view/782-thelancet-serie-sobre-lactancia-materna2016-espanol

3 Gutiérrez JP, Rivera-Dommarco J, Shamah-Levy T, Villalpando-Hernández 


\section{JOURNALOF \\ NURSING \\ M०HEALTH}

ISSN 2236 - 1987

8 Lucchini-Raies C, Márquez-Doren F, Rivera-Martínez MS. "Yo quiero amamantar a mi hijo": Develando la experiencia de mujeres que enfrentaron dificultades en su proceso de lactancia. Rev Chil Pediatr [Internet] 2017 [citado 2019 nov 30];88(5):622-8. Disponible en: https://scielo.conicyt.cl/pdf/rcp/v88 n5/art08.pdf

9 Polido CG, Mello DF, Parada CMGL, Carvalhaes MABL, Tonete VLP. Vivências maternas associadas ao aleitamento materno exclusivo mais duradouro: um estudo etnográfico. Acta Paul. Enferm. (Online). [Internet]. 2011[acesso em 2018 nov 30];24(5):624-30. Disponível em: http://www.scielo.br/pdf/ape/v24n5 /05v24n5.pdf

10 Aguilar-Cordero MJ, Baena-García L, Sánchez-López AM, Guisado-Barrilao R, Hermoso-Rodríguez $\mathrm{E}$, Mur-Villar $\mathrm{N}$. Beneficios inmunológicos de la leche humana para la madre y el niño: revisión sistemática. Nutr Hosp [Internet] 2016 [citado 2018 nov 30];33(2):482-93 Disponible en: http://scielo.isciii.es/pdf/nh/v33n2/4 6_revision3.pdf

11 Palmeira P, Carneiro - Sampaio $M$. Inmunology of breast milk. AMB rev. Assoc. Med. Bras. [Internet] 2016 [cited 2018 Nov 30];62(6):584-93. Available from: http://www.scielo.br/pdf/ramb/v62n 6/0104-4230-ramb-62-6-0584.pdf

12 Rodríguez RMR, González MGH. Tensiones y contradicciones en la intervención gubernamental para la promoción de la lactancia materna. Salud colect. [Internet]. 2017[citado 2018 nov 30];13(4):677-92. Disponible 


\section{JOURNALOF \\ AND}

NURSING
ISSN 2236 - 1987

https://journals.sagepub.com/doi/pd f/10.1177/2010105815615992

18 Muñoz-Cruz R, Rodríguez-Mármol M. Autoeficacia de la lactancia materna en mujeres primíparas de Madrid. Enfermería: cuidados humanizados [Internet]. 2017[citado 2018 nov 30];6(1):19-24. Disponible en: http://www.scielo.edu.uy/pdf/ech/v 6n1/2393-6606-ech-6-01-00019.pdf

19 Demirci J, Caplan E, Murray N, Cohen S. "I just want to do everything right:" primiparous women's accounts of early breastfeeding via an appbased diary. J Pediatr Health Care [Internet]. 2018[cited 2019 Jan 07];32(2):163-72. Available from: https://www.jpedhc.org/article/S089 1-5245(17)30374-7/fulltext

20 Belintxon-Martín $M$, Zaragüeta $M C$, Adrián MC, López-Dicastillo 0 . $\mathrm{El}$ comienzo de la lactancia: experiencias de madres primerizas. An Sist Sanit Navar [Internet]. 2011[citado 2018 nov 30];34(3):409-18. Disponible en: https://recyt.fecyt.es/index.php/ASS $\mathrm{N} /$ article/viewFile/12821/9822

21 Kronborg H, Harder I, Hall EOC. First time mothers' experiences of breastfeeding their newborn. Sexual \& reproductive healthcare [Internet]. 2015[cited 2019 Jan 07];6(2):82-7. Available from: https: / /www.sciencedirect.com/scien ce/article/abs/pii/S187757561400053 6

22 Alves VH, Padoin SMM, Rodrigues DP, Branco MBLR, Marchiori GRS, Santos MV. Percepção das nutrizes acerca do valor útil do apoio ao aleitamento materno. J. nurs. health. [Internet]. 2018[acesso em 2019 jan 07];8(3):e188306. Disponível em: 


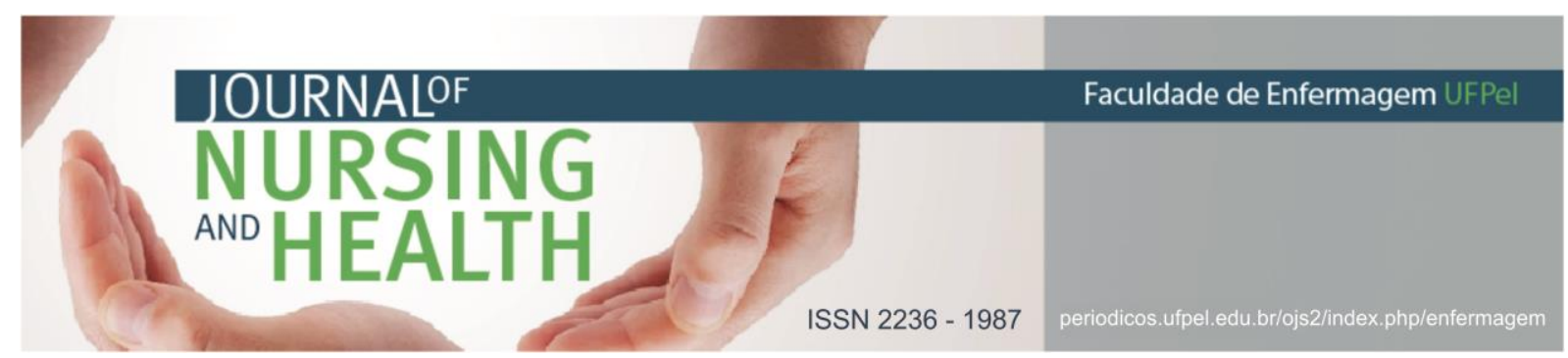

https://www.periodicos.ufpel.edu.br /ojs2/index.php/enfermagem/article /viewFile/14166/8941

Data de submissão: 19/10/2018

Data de aceite: 10/12/2018

Data de publicação: 20/02/2019 